\title{
Influence of aerosol and surface reflectance variability on hyperspectral observed radiance
}

\author{
C. Bassani ${ }^{1}$, R. M. Cavalli ${ }^{1}$, and P. Antonelli ${ }^{2}$ \\ ${ }^{1}$ Institute for Atmospheric Pollution Research (IIA) - Italian National Research Council (CNR) - Via Salaria Km. 29, \\ 300 - 00015 Monterotondo, Rome, Italy \\ ${ }^{2}$ Space Science Engineering Center, University of Wisconsin, Madison, USA \\ Correspondence to: C. Bassani (cristiana.bassani@iia.cnr.it)
}

Received: 1 October 2011 - Published in Atmos. Meas. Tech. Discuss.: 5 December 2011

Revised: 14 May 2012 - Accepted: 15 May 2012 - Published: 1 June 2012

\begin{abstract}
Current aerosol retrievals based on visible and near infrared remote-sensing, are prone to loss of accuracy, where the assumptions of the applied algorithm are violated. This happens mostly over land and it is related to misrepresentation of specific aerosol conditions or surface properties. New satellite missions, based on high spectral resolution instruments, such as PRISMA (Hyperspectral Precursor of the Application Mission), represent a valuable opportunity to improve the accuracy of $\tau_{550}^{\mathrm{a}}$ retrievable from a remote-sensing system developing new atmospheric measurement techniques. This paper aims to address the potential of these new observing systems in more accurate retrieving $\tau_{550}^{\mathrm{a}}$, specifically over land in heterogeneous and/or homogeneous areas composed by dark and bright targets. The study shows how the variation of the hyperspectral observed radiance can be addressed to recognise a variation of $\Delta \tau_{550}^{\mathrm{a}}=0.02$. The goal has been achieved by using simulated radiances by combining two aerosol models (urban and continental) and two reflecting surfaces: dark (represented by water) and bright (represented by sand) for the PRISMA instrument, considering the environmental contribution of the observed radiance, i.e., the adjacency effect. Results showed that, in the continental regime, the expected instrument sensitivity would allow for retrieval accuracy of the aerosol optical thickness at $550 \mathrm{~nm}$ of 0.02 or better, with a dark surface surrounded by dark areas. The study also showed that for the urban regime, the surface plays a more significant role, with a bright surface surrounded by dark areas providing favourable conditions for the aerosol load retrievals, and dark surfaces representing less suitable situations for inversion independently of the surroundings. However, over all,
\end{abstract}

the results obtained provide evidence that high resolution observations of Earth spectrum between 400 and $1000 \mathrm{~nm}$ would allow for a significant improvement of the accuracy of the $\tau_{550}^{\mathrm{a}}$ for anthropogenic/natural aerosols over land.

\section{Introduction}

Aerosols play a significant role in atmospheric radiative forcing by scattering and absorbing radiation and by modifying physical and radiative properties of clouds, IPCC (2007). Despite the improvements in knowledge about aerosol forcing, a great deal remains uncertain. Significant effort is being made to infer the properties of aerosol at a regional and global scale by the use of data from passive airborne and space-borne observing systems. An overview of the aerosol retrieval algorithms developed for passive space-borne sensors is presented in King et al. (1999). However, the implementation of these strategies led to operational algorithms which provide a variety of results. Recent inter-comparisons of aerosol retrievals obtained over land by different algorithms, presented in Kokhanovsky et al. (2007), have shown relatively large discrepancies between different satellite observations. Furthermore, it was shown that the methodologies used for aerosol retrieval from multispectral data, are heavily dependent on the characterisation of the a priori knowledge on aerosol models and ground surface properties.

For example, the algorithms used to determine aerosol properties over land and over ocean based on EOS-MODIS (both Terra and Aqua satellites) observed radiances, assumes that surface reflectances in the visible and near infrared are 
correlated (Kaufman et al., 1997b, 2002). Cases for which the assumptions are violated lead to lower accuracy of the retrieval products.

The challenge of imaging spectroscopy, hereafter referred to as hyperspectral remote-sensing, is to mitigate the dependency of current aerosol retrieval algorithms from these kind of assumptions, by providing observations at higher spectral and spatial resolution (Guanter et al., 2009; Gao et al., 2009; Goetz et al., 1985). However, having a single instrument in orbit represents a limitation in data availability which translates to a lack of opportunities for hyperspectral-based algorithm development and validation.

Currently, high spectral resolution data are acquired by the Compact High-Resolution Imaging Spectrometer (CHRIS) as part of the Project for On-Board Autonomy (PROBA) platform system Barnsley et al. (2004). In addition, two new hyperspectral missions namely: EnMAP (Environmental Mapping and Analysis Program), Kaufmann et al. (2008), and PRISMA (Hyperspectral Precursor and Application Mission), Galeazzi et al. (2008, 2009), have started. Both missions are intended to provide new observations at approximately $30 \mathrm{~m}$ resolution to test and improve the algorithms currently used in atmospheric studies.

There is extensive literature on the results obtained from simulated hyperspectral data (Kaufman et al., 1997a; Vermote et al., 1997; Kotchenova et al., 2008; Kokhanovsky et al., 2010). In particular, Guanter et al. (2007); Gao et al. (2009); Bassani et al. (2010) showed that with hyperspectral data, minimization algorithms can be used to solve the inverse problem to infer the aerosol optical thickness for a given aerosol model.

For this reason, the aim of this study is to investigate the influence of the aerosol model, the surface properties and the adjacency effect on the accuracy of the aerosol optical thickness at $500 \mathrm{~nm}$ retrieval from hyperspectral observations. The whole study was done on simulated data and was divided in two parts. The first part was based on an ideal instrument with $2.5 \mathrm{~nm}$ spectral resolution, and the second part on PRISMA-like data obtained convolving the ideal instrument data with an instrument Gaussian response function with Full Width at Half Maximum (FWHM $\leq 10 \mathrm{~nm}$ ) and spectral coverage between $400-1000 \mathrm{~nm}$.

Aerosol properties considered in this study were: aerosol loading at optical thickness of $550 \mathrm{~nm}$, referred to as $\tau_{550}^{\mathrm{a}}$, and aerosol model (urban or continental). The analysis was performed on a dark (clear-water) and a bright (sand) surface to evaluate the radiative impact of the reflective characteristics of the target on the simulated radiances, which also accounted for the surrounding environment contribution. Synthetic radiances were simulated using the 6SV1.1 version of the forward model 6SV (Second Simulation of a Satellite Signal in the Solar Spectrum - Vector) (Vermote et al., 2006).

The first specific goal was to provide qualitative analysis of how observed radiance depend on aerosol optical thickness $\tau_{550}^{\mathrm{a}}$ at $500 \mathrm{~nm}$, on aerosol models over land (continental and urban) and on target surface reflective properties (including target surroundings). Analysis was based on simulated radiances at $2.5 \mathrm{~nm}$ resolution for the spectral region of 400 $2500 \mathrm{~nm}$.

The second specific goal was to identify optimal conditions at high spatial resolution, in terms of target and surrounding surface reflective characteristics, for anthropogenic/natural aerosol property retrievals. For this part, simulated data were tailored to PRISMA instrument specifications. The goal was achieved by comparing the sensitivity of the simulated radiances to incremental changes in aerosol optical thickness of 0.02 , with the signal-to-noise ratio specified for the PRISMA instrument, for different target areas.

The methodology followed in the study is described in detail in Sect. 2, while Sect. 3 contains the general results obtained for an idealized hyperspectral instrument, and Sect. 4 focuses on the specific results obtained for a PRISMA-like instrument, with the conclusions dedicated to the closing section.

\section{Methodology}

This section aims to introduce the scientific methodology followed in the study. In both cases, simulated radiances accounted explicitly for the adjacency effect, i.e., the impact of the surroundings of the target area on the observed radiances. The analysis of the environmental contribution was taken into account to address whether the environment contributions play a significant role in the accuracy of the retrieved aerosol optical thickness or not.

This section is divided into three subsections; the first is dedicated to the theoretical aspects of the simulation of observed radiances for an ideal instrument, the second is focused on the contribution of the aerosol loading and models on the simulated data, and the last one is dedicated to the surface contribution to the simulated radiances.

\subsection{Observation simulation}

Synthetic radiances used for this study, were generated within the spectral domain of $400-2500 \mathrm{~nm}$ sample at $2.5 \mathrm{~nm}$, for different values of aerosol optical thickness at $550 \mathrm{~nm}, \tau_{550}^{\mathrm{a}}$ and for urban and continental models. The model used in the simulation is based on the equation for the top of the atmosphere radiance presented in Vermote et al. (1997), which considers the anisotropy negligible of the reflecting surface, (assumption of Lambertian surface):

$$
L_{\mathrm{v}}(\lambda)=\frac{\mu_{\mathrm{s}} E_{\mathrm{s}}(\lambda)}{\pi} t^{\mathrm{g}}(\lambda)\left[\rho^{\mathrm{atm}}(\lambda)+\frac{T^{\uparrow}(\lambda) T^{\downarrow}(\lambda) \rho_{\mathrm{gnd}}(\lambda)}{1-S(\lambda) \rho_{\mathrm{gnd}}(\lambda)}\right]
$$

where $L_{\mathrm{V}}(\lambda)$ is the radiance by the ideal sensor within the considered Field Of View (FOV); $E_{\mathrm{S}}(\lambda)$ is the solar irradiance at the Top Of Atmosphere (TOA); $t^{\mathrm{g}}(\lambda)$ is the transmittance due to gaseous absorption; $\rho^{\text {atm }}(\lambda)$ is the intrinsic 
Table 1. The volumetric percentage of the four basic components (oceanic, water-soluble, soot and dust-like) describing the urban and continental aerosol model, d'Almeida et al. (1991).

\begin{tabular}{lcccc}
\hline & Water-soluble & Soot & Dust-like & Oceanic \\
\hline Urban & $61 \%$ & $22 \%$ & $17 \%$ & $0 \%$ \\
Continental & $29 \%$ & $1 \%$ & $70 \%$ & $0 \%$ \\
\hline
\end{tabular}

atmospheric reflectance; $T^{\uparrow}(\lambda)=e^{-\tau(\lambda) / \mu_{\mathrm{v}}}+t_{\mathrm{d}}\left(\mu_{\mathrm{v}}, \lambda\right)$ and $T^{\downarrow}(\lambda)=e^{-\tau(\lambda) / \mu_{\mathrm{s}}}+t_{\mathrm{d}}\left(\mu_{\mathrm{s}}, \lambda\right)$ are the total upwelling and downwelling transmittance, both with direct, $e^{-\tau(\lambda) / \mu_{\mathrm{s}, v}}$, and diffuse, $t_{\mathrm{d}}\left(\mu_{\mathrm{s}, \mathrm{v}}, \lambda\right)$, components; $\mu_{\mathrm{s}, \mathrm{v}}=\cos \left(\theta_{\mathrm{s}, \mathrm{v}}\right)$ where $\theta_{\mathrm{s}, \mathrm{v}}$ are the solar, "s", and view, "v", zenith angle; $\tau(\lambda)$ is the atmospheric optical thickness; $S(\lambda)$ is the atmospheric spherical albedo, defined in Kokhanovsky (2008), and $\rho_{\text {gnd }}(\lambda)$ is the at-ground surface reflectance.

Simulated radiances were obtained using the last version (v. 6SV1.1, Kotchenova et al., 2008) of the Second Simulation of a Satellite Signal in the Solar Spectrum (6S) radiative transfer code Vermote et al. (1997). The 6SV1.1 is an opensource code which implements Eq. (1). It shows significant improvements with respect to previous versions, as described in Vermote and Kotchenova (2009).

Simulation of the radiance observed by the ideal instrument were obtained accounting not only for the radiative contribution of the viewed target, but also for the contribution of areas surrounding the target FOV (Field Of View), due to scattering processes. This second contribution is generally referred to as the adjacency effect. The equation used to calculate the observed radiance which accounts for the environmental contribution was presented by Vermote et al. (1997)

$$
\begin{aligned}
& L(\lambda)=\frac{\mu_{\mathrm{s}} E_{\mathrm{s}}(\lambda)}{\pi} t^{\mathrm{g}}(\lambda) \\
& {\left[\rho^{\operatorname{atm}}(\lambda)+\frac{T^{\downarrow}(\lambda) e^{-\tau / \mu_{\mathrm{v}}} \rho_{\mathrm{gnd}}(\lambda)+T^{\downarrow}(\lambda) t_{\mathrm{d}}\left(\mu_{\mathrm{v}}\right)<\rho_{\mathrm{gnd}}(\lambda)>}{1-S(\lambda)<\rho_{\text {gnd }}(\lambda)>}\right]}
\end{aligned}
$$

where $L(\lambda)$ is the total observed radiance coming from the considered FOV and its surroundings and $<\rho_{\text {gnd }}(\lambda)>$ represents the mean of the environmental reflectance around the viewed target. When the neighbouring targets are equal to the viewed target $\left(<\rho_{\text {gnd }}(\lambda)>=\rho_{\text {gnd }}(\lambda)\right)$, Eqs. (2) and (1) become identical.

\subsection{Aerosol contribution}

Aerosol contribution to observed radiances is mostly determined by the radiation extinction due to scattering and absorption. Aerosol loading is the aerosol primary quantity in driving radiation extinction within the atmospheric window in the visible spectral domain, and it is generally parameterized by its optical thickness at $550 \mathrm{~nm}$, referred to as $\tau_{550}^{\mathrm{a}}$. The sensitivity study presented in this paper is based on radiances simulated for:

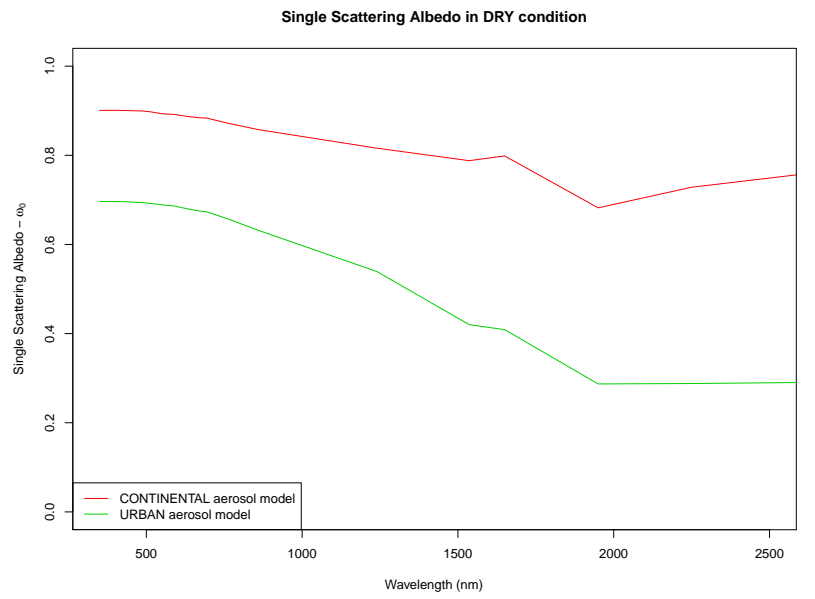

Fig. 1. Single scattering albedo $\left(\omega_{0}\right)$ of continental and urban aerosol model, available in dry condition from the 6SV1.1 code.

- $\tau_{550}^{\mathrm{a}} \in\{0.00-2.00\}$ at intervals of $\Delta \tau_{550}^{\mathrm{a}}=0.02$. The increment $\Delta \tau_{550}^{\mathrm{a}}$ was set to 0.02 to allow for direct calculation of the radiance gradient $\frac{\Delta L}{\Delta \tau_{550}^{a}}$ with the same accuracy level of in situ observation provided by sun skyradiometer, as CIMEL Holben et al. (1998), generally used for the validation of the remote aerosol retrievals. It is worth mentioning that also aerosol retrievals provided by MODIS have an expected error of $\pm\left(0.05+0.15 \tau_{550}^{a}\right)$ over land, as described in Levy et al. (2010).

Range of aerosol optical thicknesses used in the simulation was the widest allowed by 6SV1.1. Furthermore, it is the same range used to generate the main-group elements for $\tau_{550}^{\mathrm{a}}$ in the retrieval algorithm applied to hyperspectral remotely data, Guanter et al. (2007); Bassani et al. (2010). Values of $\tau_{550}^{\mathrm{a}}$ outside of this domain must be treated with different atmospheric radiative transfer codes, as in the L-POM model presented in Alakian et al. (2008), which enables the simulation of the radiative field in very high aerosol loading conditions.

- Both urban and continental aerosol models to verify the role of the optical and microphysical properties of the aerosol in the direct and diffuse components of the solar beam during its propagation through the atmosphere in a homogeneous and heterogeneous environment composed by dark and/or bright target. The present study focuses on the two models which are mostly used in remote data acquired over land. Both models combine the four basic components: oceanic, water-soluble, soot and dust-like, d'Almeida et al. (1991). Table 1 shows the volumetric percentage of the basic components for the urban and continental aerosol regimes, as contained in the source code 6SV1.1. Figure 1 shows the single scattering albedo in dry condition for both the aerosol models, like available from the 6SV1.1 code. 


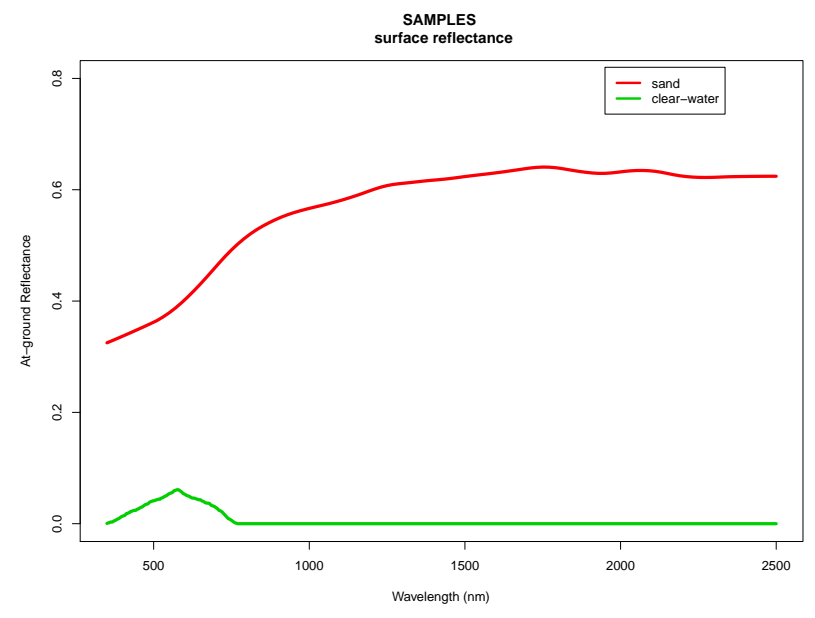

Fig. 2. Surface reflectance, $\rho_{\text {gnd }}(\lambda)$ contained in the 6SV1.1 source code: sand for bright and water for dark in the spectral domain: 400 $2500 \mathrm{~nm}$ sampled at $2.5 \mathrm{~nm}$.

\subsection{Surface contribution}

To properly simulate observed radiances for the presented study, the influence of the surface contributions was explicitly taken into consideration. The interaction between the radiation and the surface affects both the direct and diffuse components coming off the viewed target and the diffuse component from adjacent targets which is, successively, scattered from the atmospheric aerosols.

Simulation of observed radiances, generated for this study, satisfies the Lambertian condition, required by Eqs. (1) and (2). Therefore, the $\rho_{\text {gnd }}(\lambda)$, in order to satisfy the previous requirement, were selected for sand (representative of a bright surface) and water (representative of a dark surface). Figure 2 shows the two reflectance spectra used by 6SV1.1 for the analysis. The two spectral signatures were used for the viewed and adjacent target. Table 2 provides the naming convention for the radiance generated by combining the two spectral signatures for the viewed and adjacent targets.

\section{General results for idealized instrument}

This section shows the results obtained by investigating the dependency of the observation simulated according to Sects. 2.1, 2.2 and 2.3 for different combinations of aerosol and surface properties. The simulation was performed in dry condition. In this way, it was possible to neglect the hygroscopic properties of the aerosol models that can change the radiative effects of both the aerosol models on the observed radiance. The geometrical conditions used in the simulation were chosen to maximise the upwelling solar irradiance reflected by the surface. The FOV was located in Rome (Latitude: $41^{\circ} 58^{\prime} \mathrm{N}$, Longitude: $12^{\circ} 40^{\prime} \mathrm{E}$ ), Italy. The acquisition time was assumed midday in July with the so-
Table 2. The observed radiance simulated using sand (representative for bright) and water (representative for dark) for viewed and adjacent targets.

\begin{tabular}{ccc}
\hline Observed radiance & Viewed target & Adjacent targets \\
\hline$L^{\mathrm{ss}}(\lambda)$ & sand & sand \\
$L^{\mathrm{sw}}(\lambda)$ & sand & clear-water \\
$L^{\mathrm{ws}}(\lambda)$ & clear-water & clear-water \\
$L^{\mathrm{ww}}(\lambda)$ & clear-water & sand \\
\hline
\end{tabular}

lar zenith angle of $\theta_{\mathrm{s}}=33.97$ and the azimuth solar angle of $\phi_{\mathrm{s}}=238.41$. The nadir viewing angle was chosen to verify the symmetry in azimuth of the environment contribution (adjacency effect) on the observed radiance, as shown in Fig. 5 of Vermote et al. (1997).

\subsection{The impact of aerosol loading on the observed radiance}

As a first step, the analysis was conducted, not taking into account the environmental contribution or adjacency effect, to the observed radiances. In order to better characterise the variation of the observed radiance, $L(\lambda)$, as function of the increasing of $\tau_{550}^{\mathrm{a}}$ the quantity $\delta L=\left(L(\lambda)-L_{0}(\lambda)\right) / L(\lambda)$ was used to present the results obtained for each simulation. $L_{0}(\lambda)$ is the observed radiance in the absence of aerosol, $\tau_{550}^{\mathrm{a}}=0$, and the $L(\lambda)$ follows the configurations expressed in the first column of Table 2.

Figure 3 shows the results obtained by simulating the radiance for the aerosol optical thickness, $\tau_{550}^{\mathrm{a}} \in 0.00-2.00$ with $\Delta \tau_{550}^{\mathrm{a}}=0.1$. The variation in simulated radiance due to increasing aerosol loading was determined for both urban and continental models. Under high surface reflectance, for sand-type FOVs (top images in Fig. 3), the attenuation of radiance, which occurs with the increase in aerosol optical thickness at $550 \mathrm{~nm}$, is more evident for the urban (more absorbing) aerosol model than for the continental (less absorbing) model. The spectral behaviour associated with the dark surface, water FOVs (bottom images in Fig. 3), was the opposite of the one associated with the bright surfaces. Over dark surfaces, observed radiance tends to increase with the increasing of the aerosol loading, especially for continental aerosol, which are less absorbing than the urban ones, as reported also in Seidel and Popp (2011).

Results obtained for an idealized hyperspectral instrument, neglecting the adjacency effect, show that the higher sensitivity of the observed radiance for bright surfaces, makes them well suited for aerosol retrievals both in presence of anthropogenic (urban) pollution, or continental-type of aerosols. Dark surfaces, in case of continental aerosols, still provide good conditions for retrievals, while in presence of urban aerosols the low radiance sensitivity to variation of the particulate loading make this kind of target not well-suited for inversion. 

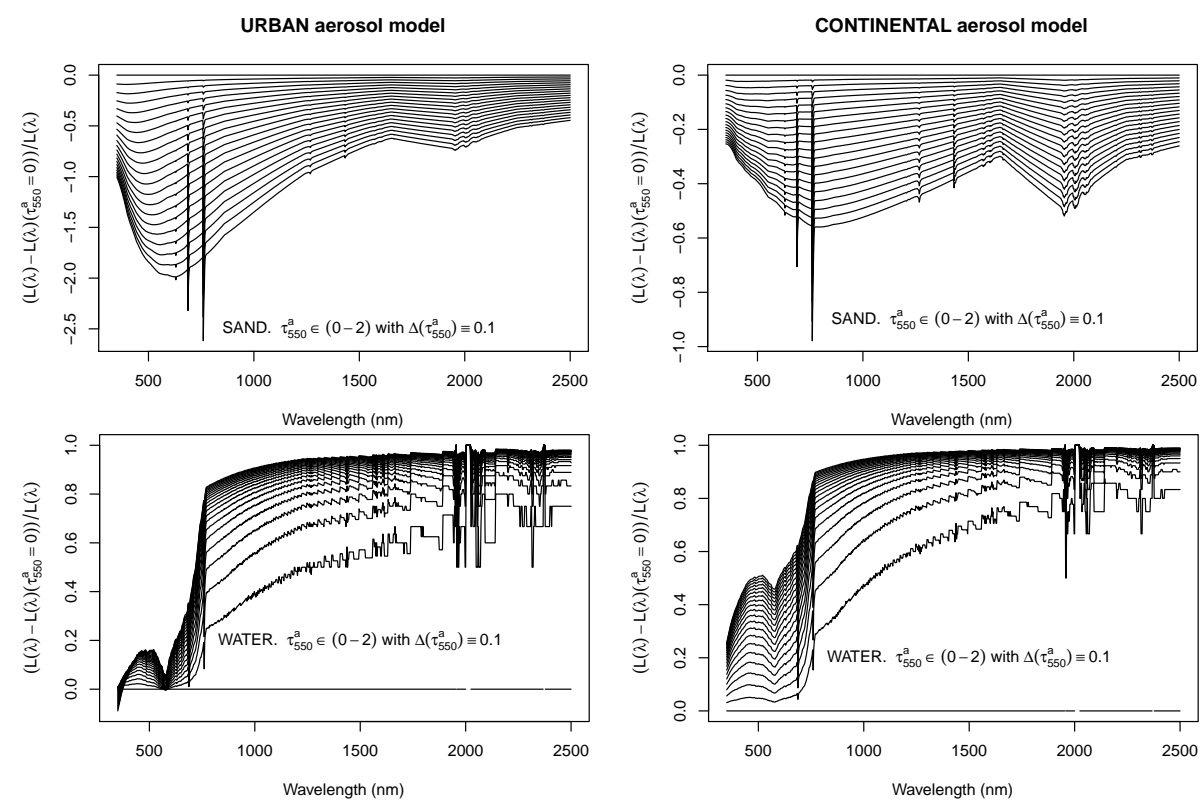

Fig. 3. $\delta L=\left(L(\lambda)-L_{0}(\lambda)\right) / L(\lambda)$ with $\tau_{550}^{\mathrm{a}} \in 0.0,2.0$ with $\Delta \tau_{550}^{\mathrm{a}}=0.1$ using the urban (left column) and continental (right column) aerosol model for sand (first row) and water (second row).

\subsection{The impact of aerosol loading and the environment on the observed radiance}

Results obtained in Sect. 3.1 were refined by introducing the environmental contribution (adjacency effect) in the simulated radiances. The analysis, taking into account the diffuse component to the observed radiance coming from the surrounding environment, addresses the impact of the adjacency effect on the sensitivity of the observed radiance to the $\tau_{550}^{\mathrm{a}}$. The analysis of the effect of the 4 possible combinations target-surroundings (Table 2 ) on the simulated radiance, $L(\lambda)$, was performed by numerical evaluation of Eq. (2) where $<\rho_{\text {gnd }}(\lambda)>$ was calculated off the two surface reflectance spectra presented in Fig. 2. Two of the possible four combinations, namely $L^{\mathrm{ss}}(\lambda)$ and $L^{\mathrm{ww}}(\lambda)$ represent homogeneous situations, while $L^{\mathrm{sw}}(\lambda)$ and $L^{\mathrm{ws}}(\lambda)$ represent heterogeneous conditions.

\subsubsection{Bright-target}

Figure 4 shows the simulation of the sand FOV with $\tau_{550}^{\mathrm{a}} \in$ $0.00-2.00$ sampled at $\Delta \tau_{550}^{\mathrm{a}}=0.1$ in the homogeneous condition (top images), $\delta L^{\mathrm{ss}}(\lambda)$, and in the heterogeneous scene (bottom images), $\delta L^{\mathrm{sw}}(\lambda)$. The two columns represent the individual results for the urban model (left) and the continental model (right).

Results show that the observed radiance is more sensitive (high values of $\delta L$ ) to the increase of the aerosol loading when surrounded by a dark environment in urban and continental model. This behaviour is more evident for bright targets surrounded by dark areas in urban model (lower left image in Fig. 4). While for a bright target surrounded by a bright environment, $\delta L$ shows no significant differences between radiances obtained with and without adjacency effect, for continental and urban regimes (top images of Fig. 3). The sensitivity of $\delta L$, benefits from the diffuse component when its contribution is low, with respect to the direct component coming from the observed target. Thus, the sensitivity of the observed radiance to the aerosol optical thickness, in the bright-target case appears to increase for a dark environment for both aerosol models. In particular, the dark environment is weaker in an urban regime, where the absorbing aerosol decreases the observed radiance making the bright-case in a heterogeneous environment the best condition for accurate aerosol retrieval by using hyperspectral remote-sensing data.

\subsubsection{Dark-target}

Figure 5 shows the simulation for water FOV with $\tau_{550}^{\mathrm{a}} \in$ $0.00-2.00$ sampled at $\Delta \tau_{550}^{\mathrm{a}}=0.1$ in the heterogeneous condition (top images), $\delta L^{w s}(\lambda)$, and in homogeneous conditions (bottom images), $\delta L^{\mathrm{ww}}(\lambda)$. The two columns represent the individual results for the urban model (left) and the continental model (right).

Plots in the left column highlight the spectral regions in which the absorption of the urban aerosol plays a crucial role in the extinction of radiation. In these regions, mostly in the visible, it is evident that the observed radiance for a dark target is weakly dependent on the aerosol load increase. Therefore, the $\tau_{550}^{\mathrm{a}}$ retrieval, in the presence of strong absorbing aerosols (urban) over dark targets, results in being very challenging due to the limited sensitivity (see Fig. 5 lower 

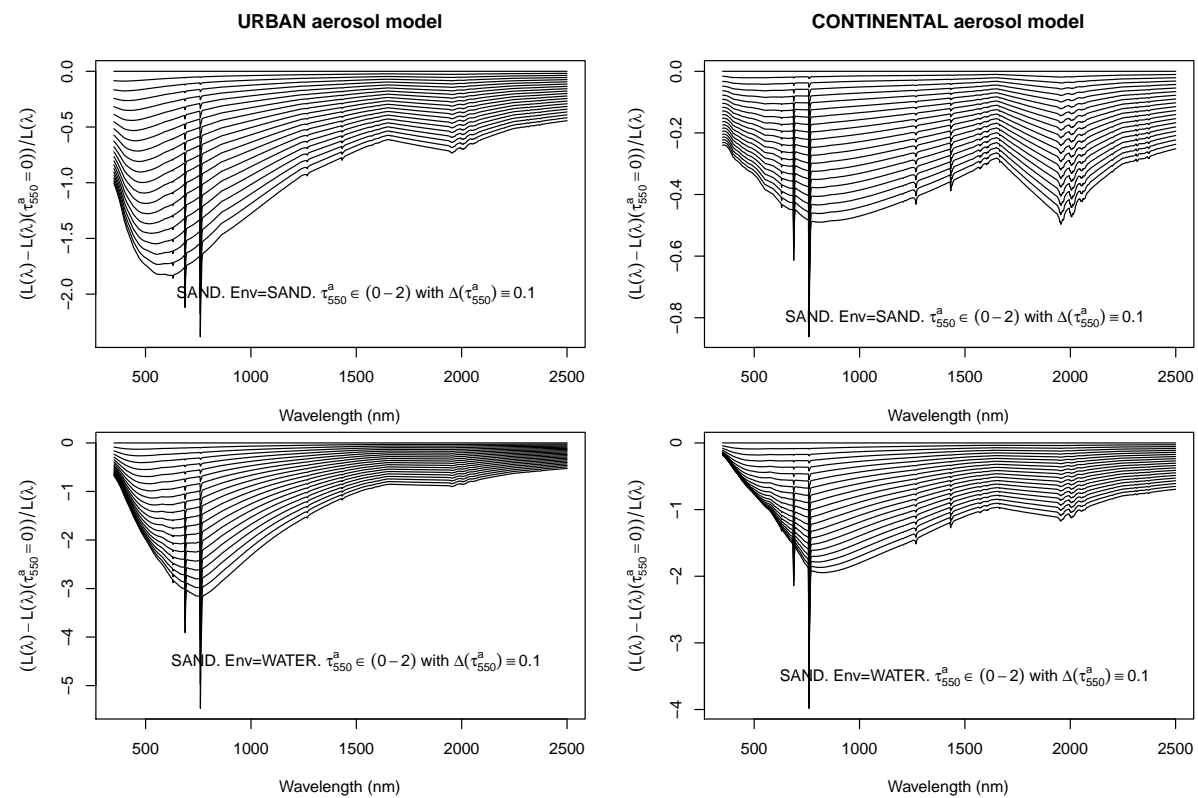

Fig. 4. $\delta L=\left(L(\lambda)-L_{0}(\lambda)\right) / L(\lambda)$ with $\tau_{550}^{\mathrm{a}} \in 0.0,2.0$ with $\Delta \tau_{550}^{\mathrm{a}}=0.1$ using the urban (first column) and continental (second column) aerosol models to view sand surrounded by sand, $\delta L^{\mathrm{ss}}(\lambda)$ (first row) and water, $\delta L^{\mathrm{sw}}(\lambda)$ (second row).
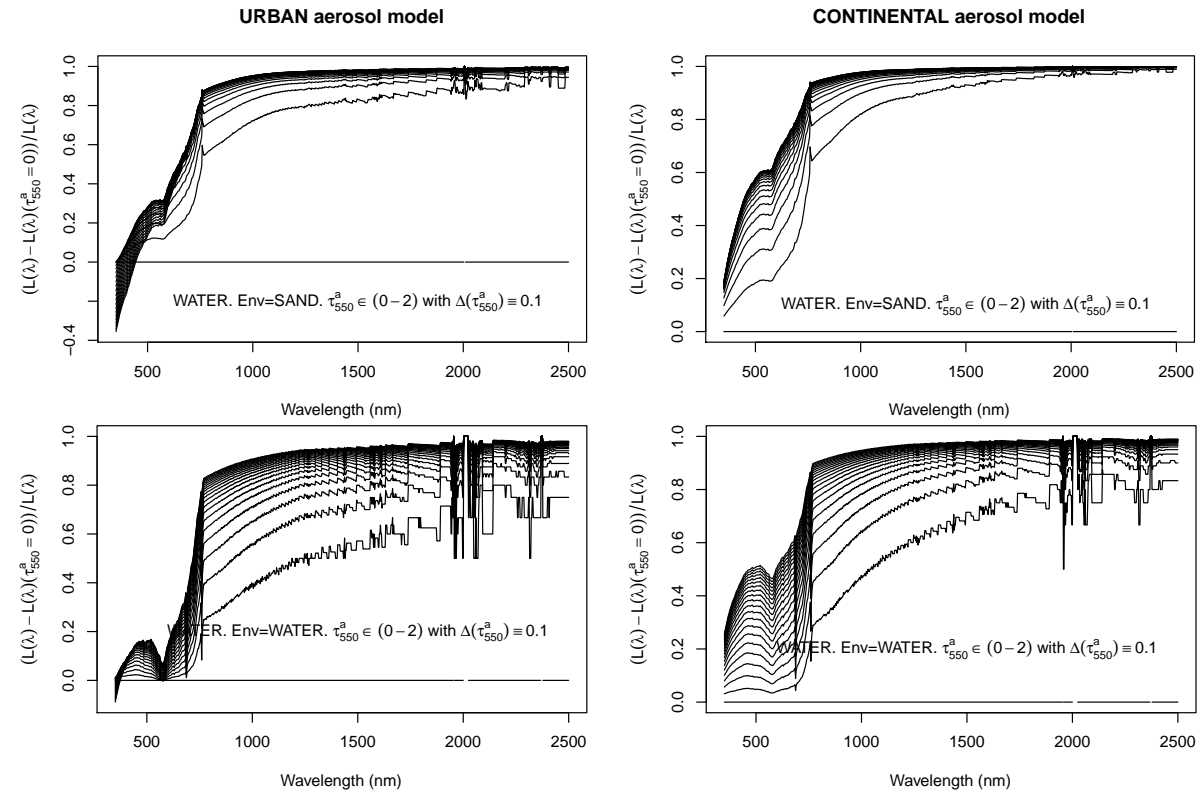

Fig. 5. $\delta L=\left(L(\lambda)-L_{0}(\lambda)\right) / L(\lambda)$ with $\tau_{550}^{\mathrm{a}} \in 0.0,2.0$ with $\Delta \tau_{550}^{\mathrm{a}}=0.1$ using the urban (first column) and continental (second column) aerosol models to view water surrounded by sand, $\delta L^{\mathrm{ws}}(\lambda)$ (first row) and water, $\delta L^{\mathrm{ww}}(\lambda)$ (second row).

left panel). Whereas, the plots on the right column show that variations in the observed radiance in the continental regime are more evident when the dark target is surrounded by dark areas. In this case, for $\tau_{550}^{\mathrm{a}} \in\{0.00,2.00\}$ the signal at the sensor increases with the increasing of the aerosol load ( $\delta L$ is greater than 0 ). Radiances increase rapidly for $0<\tau_{550}^{\mathrm{a}}<1$, while for $\tau_{550}^{\mathrm{a}}>1$, dependency on the aerosol load is definitely weaker.
Thus, it should be expected that aerosol retrieval using hyperspectral data over water is more sensitive in continental regimes than in the urban ones. Besides, water surface provides a better suited target for accurate aerosol retrievals when the FOV is surrounded by a dark environment. The low values of the observed radiance coming directly from the observed dark target are crucial for the sensitivity of the observed radiance. In fact, $\delta L$ does not show any gain 
from the surrounding contribution even in the case of dark environment. Therefore, the optimal conditions for accurate retrievals are dark targets surrounded by dark environment. Analysis of $\delta L$ shows that the observed radiance are equally sensitive to aerosol load with and without the adjacency effect, for continental and urban regimes (bottom images of Fig. 3).

\section{Specific results for PRISMA-like}

While the first part of the presented study is focused on the sensitivity of an ideal hyperspectral sensor to aerosol retrieval, this part aims to investigate, in detail, the potential use of PRISMA (Hyperspectral Precursor of the Application Mission) for accurate aerosolic retrievals with $\Delta \tau_{550}^{\mathrm{a}}=0.02$ and to demonstrate limits and benefits of the environmental contribution on the observed radiance acquired from a specific sensor for aerosol retrieval accuracy. PRISMA is a medium-resolution hyperspectral imaging instrument developed under the guidance of the Italian Space Agency (ASI) Galeazzi et al. (2008). Instrument specifications are listed in Table 3.

Radiances simulated according to Sects. 2.1, 2.2 and 2.3, in the 400-2500 $\mathrm{nm}$ were convolved to the Gaussian response function of the PRISMA channels belonging to the spectral domain where the aerosol and environment effects are relevant and cause different behaviours of the observed radiance between a dark-target and a bright-target case, that is 400$1000 \mathrm{~nm}$. From the Table 3, all the 92 channels of the first spectrometer fall into this spectral domain.

To assess the value of PRISMA in retrieving aerosol loadings, the ratio $\left.\frac{L(\lambda)}{\Delta L(\lambda)}\right|_{\tau_{550}^{\mathrm{a}}}$, where $\Delta L(\lambda)$ is the variation of radiance due to a 0.02 increment in aerosol optical thickness, was compared to the minimum Signal-to-Noise Ratio $(\mathrm{SNR} \approx 200)$ specified for the instrument and listed in Table 3. Cases (combinations of aerosol and surface contributions) associated to $\left.\frac{L(\lambda)}{\Delta L(\lambda)}\right|_{\tau_{550}^{\mathrm{a}}}<200$ were considered invertible by PRISMA-tailored retrieval algorithm.

While the surface properties and the aerosol modes were kept identical to those of Sect. 3 (only sand and water targets were considered in urban and continental regimes), the aerosol loadings were varied at incremental steps of $\Delta \tau_{550}^{\mathrm{a}}=$ 0.02 with the goal to identify, with a quantitative analysis, the conditions and the spectral regions for which the radiance sensitivity to aerosol loading changes is greater than the specification for the instrument SNR (i.e., $\frac{L(\lambda)}{\Delta L(\lambda)}<$ SNR).

\subsection{The impact of aerosol loading on the PRISMA radiance}

Figure 6 shows the ratio $\frac{L(\lambda)}{\Delta L(\lambda)}$ (in greyscale) for the observed radiances of a PRISMA-like instrument, calculated for aerosol loading $\tau_{550}^{\mathrm{a}} \in\{0.00,2.00\}$ with $\Delta \tau_{550}^{\mathrm{a}}=0.02$ (on the $y$-axis) and $\lambda$ in the range $400-1000 \mathrm{~nm}$ (on the x-axis).
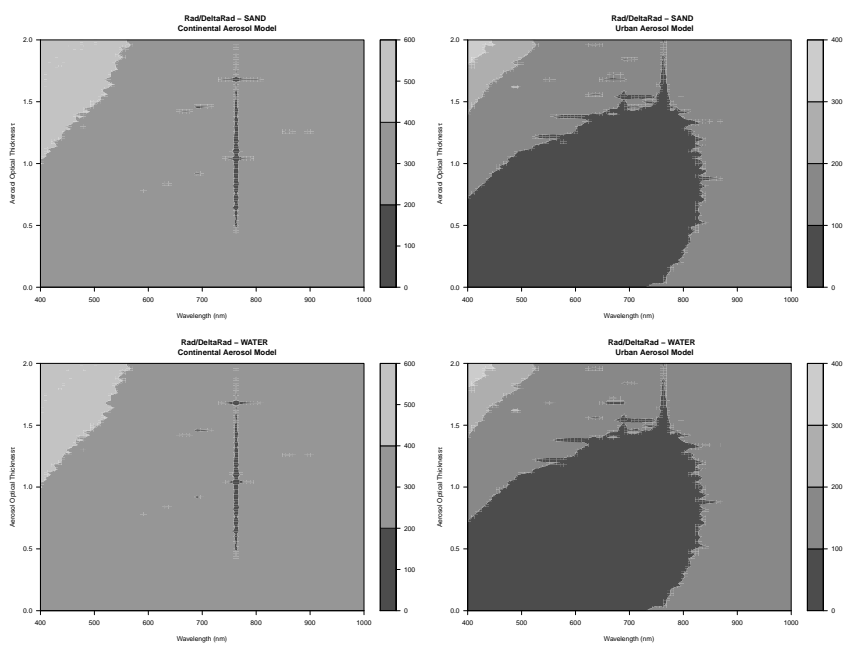

Fig. 6. The $\frac{L(\lambda)}{\Delta L(\lambda)}$ in the PRISMA observed radiance simulated by increasing the aerosol loading, $\tau_{550}^{\mathrm{a}} \in\{0.00-2.00\}$ with $\Delta \tau_{550}^{\mathrm{a}}=$ 0.02 , for sand (first row) and water (second row) in continental (first column) and urban (second column) aerosol model.

Results, obtained without taking into account the adjacency effect, show that radiance sensitivity is greater than instrument SNR only for urban aerosolic regimes (left column) for both bright and dark targets. For continental regimes (left column), independently on the reflective characteristics of the surface, $\frac{L(\lambda)}{\Delta L(\lambda)}$ was found to be smaller than the SNR only in narrow absorption bands.

Thus, the quantitative analysis proved that, in urban regimes, PRISMA would provide observations well suited for aerosol optical thickness retrieval especially for $\lambda<$ $600 \mathrm{~nm}$ independently on the surface reflective properties. In addition, retrievals, always in urban regimes, are expected to be more accurate for values of $\tau_{550}^{\mathrm{a}}<1.5$, where the instrument sensitivity to changes in the aerosol load is highest. Continental regimes represent cases where the inversion might require a pre-processing of the observation (such as PCA analysis) to increase the SNR.

Figure 6 and the following figures, show the surface of the dependence of the observed radiance to the aerosol loading like function of $\frac{L(\lambda)}{\Delta L(\lambda)}$. The wiggly structures of the figures are caused by the interpolation of the simulated values and they have not a physical origin.

\subsection{The impact of aerosol and environment on the PRISMA radiance}

Results obtained in Sect. 4.1 were refined by including environmental contribution (adjacency effect). The analysis of the effect of the 4 possible combinations target-surroundings (Table 2) on the simulated radiance, $L(\lambda)$, was performed by numerical evaluation of Eq. (2), where $<\rho_{\text {gnd }}(\lambda)>$ was calculated off the two surface reflectance spectra presented in Fig. 2. Two of the possible four combinations, namely $L^{\mathrm{ss}}(\lambda)$ 
Table 3. PRISMA instrument specifications, available from the website: http://www.asi.it/files/The\%20PRISMA\%20mission.pdf.

\begin{tabular}{ccc}
\hline Parameter & $\begin{array}{c}\text { I Spectrometer } \\
\text { VNIR }\end{array}$ & $\begin{array}{c}\text { II Spectrometer } \\
\text { SWIR }\end{array}$ \\
\hline Spectral range & $400-1010 \mathrm{~nm}$ & $920-2505$ \\
Spectral resolution (FWHM) & $\leq 10 \mathrm{~nm}$ & $\leq 12 \mathrm{~nm}$ \\
Spectral bands & 92 & 171 \\
Swath width (Km) & 30 & 30 \\
Ground sample distance (m) & 30 & 30 \\
\hline SNR (Signal-to-Noise Ratio) & $\geq 200$ in the range $0.4-1.0 \mu \mathrm{m}$ & $\geq 200$ in the range $1.00-1.75 \mu \mathrm{m}$ \\
& 600 at $0.65 \mu \mathrm{m}$ & $>400$ at $1.55 \mu \mathrm{m}$ \\
& & $\geq 100$ in the range $1.95-2.35 \mu \mathrm{m}$ \\
& & $>200$ at $2.1 \mu \mathrm{m}$ \\
\hline
\end{tabular}

and $L^{\mathrm{ww}}(\lambda)$ represent homogeneous situations, while $L^{\mathrm{sw}}(\lambda)$ and $L^{\mathrm{ws}}(\lambda)$ represent heterogeneous conditions.

Figure 7 shows the ratio $\frac{L(\lambda)}{\Delta L(\lambda)}$ (in greyscale) as a function of aerosol loading ( $\mathrm{y}$-axis) and wavelength ( $\mathrm{x}$-axis) for bright target and bright (top) and dark (bottom) environmental reflectance. Top images (compared to Fig. 6) show that the adjacency effect for homogeneous conditions do no improve the instrument sensitivity for the continental regime (left column), neither for the urban regime (right column). In case of heterogeneous conditions, both for continental (bottom left) and for the urban (bottom right), the dark environment significantly improves the instrument sensitivity (by reducing the ratio $\left.\frac{L(\lambda)}{\Delta L(\lambda)}\right)$. It is worth mentioning that the heterogeneous environment (water surroundings) for bright targets, could improve the accuracy of retrieved $\tau_{550}^{\mathrm{a}}$ by enabling inversion algorithms to use more channels (as the spectral range where $\frac{L(\lambda)}{\Delta L(\lambda)}<$ SNR is broader). Therefore, PRISMA-like data are well suited to infer aerosolic properties, especially in heterogeneous regions, under urban conditions.

Figure 8 shows the ratio $\frac{L(\lambda)}{\Delta L(\lambda)}$ (in greyscale) as a function of aerosol loading ( $\mathrm{y}$-axis) and wavelength ( $\mathrm{x}$-axis) for dark targets and bright (top) and dark (bottom) environmental reflectance. Top images (compared to Fig. 6) show that the adjacency effect for heterogeneous conditions do improve the instrument sensitivity for the continental regime (left column) neither for the urban regime (right column). In case of homogeneous conditions, for continental (bottom left), the dark environment improves the instrument sensitivity (by reducing the ratio $\frac{L(\lambda)}{\Delta L(\lambda)}$ ) over a broad spectral region. It can be concluded that the sensitivity of the PRISMA-like sensor to the aerosol loading, $\tau_{550}^{\mathrm{a}}$, in the continental aerosol regime represent optimal retrieval conditions when the target surroundings are dark. For urban aerosol model, the variation of the dependence of radiances on changes in aerosol optical thickness, when viewing a dark surface does not seem to be well suited for accurate retrievals. Therefore, PRISMA observations over land for continental aerosolic regimes (generally non-anthropogenic) would provide more sensitivity to the aerosol loading of observed radiance when observ-
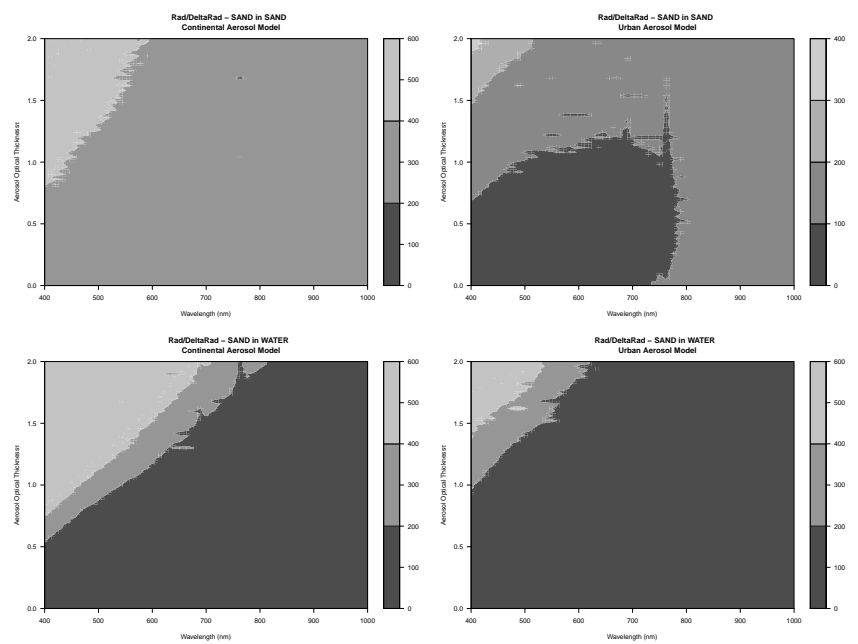

Fig. 7. The $\frac{L(\lambda)}{\Delta L(\lambda)}$ in the PRISMA observed radiance simulated by increasing the aerosol loading, $\tau_{550}^{\mathrm{a}} \in\{0.00-2.00\}$ with $\Delta \tau_{550}^{\mathrm{a}}=$ 0.02 , for the sand surface given the environmental contribution of sand (first row) and water (second row) under continental (first column) and urban (second column) aerosol model.

ing dark surface in homogeneous conditions than the bright surface.

Table 4 summarizes the best viewing conditions for inversion of PRISMA-like data over homogeneous or heterogeneous regions. For bright targets, the heterogeneous environment enhances the sensitivity of the radiances to the aerosol load, while for dark targets the environmental contribution has an impact on the sensitivity for homogeneous surroundings, and negative impact for heterogeneous conditions.

In order to verify the dependence of the results from the viewing geometry, the calculations done for the maximal solar irradiance intensity $\left(\theta_{\mathrm{s}}=33.97^{\circ}\right)$ were repeated by choosing a low irradiance case (i.e., high solar zenith angle, $\theta_{\mathrm{s}}=66.26^{\circ}$ ). Figure 9 highlights that a bright-target surrounded by a dark environment in urban aerosol regime and a dark-target surrounded by a dark environment in continental 

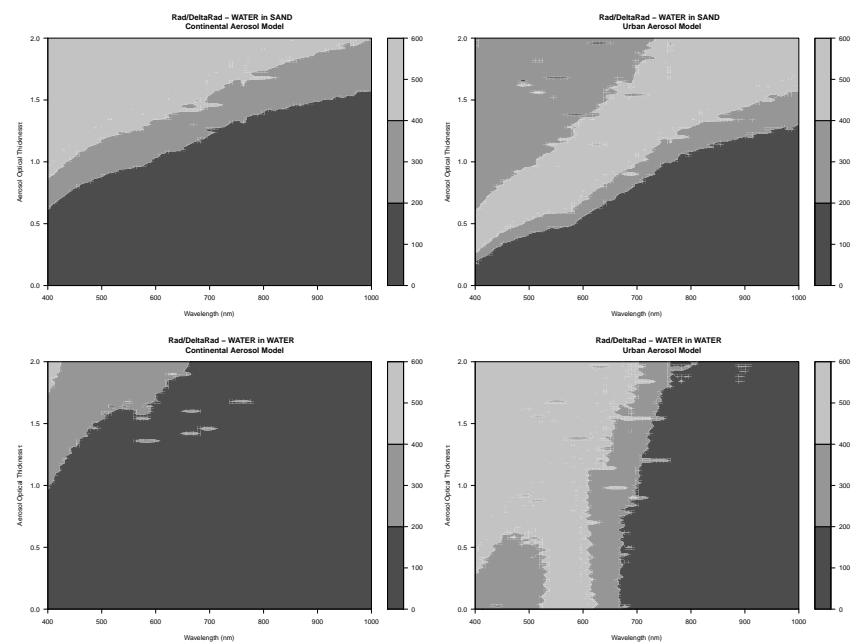

Fig. 8. The $\frac{L(\lambda)}{\Delta L(\lambda)}$ in the PRISMA observed radiance simulated by increasing the aerosol loading, $\tau_{550}^{\mathrm{a}} \in\{0.00-2.00\}$ with $\Delta \tau_{550}^{\mathrm{a}}=$ 0.02 , for water surrounded by sand (first row) and water (second row) under continental (first column) and urban (second column) aerosol model.

Table 4. Condition for accurate aerosol retrieval via PRISMA-like data.

\begin{tabular}{lcc}
\hline Aerosol model & Viewed target & Environment \\
\hline Urban & Sand & Water \\
Continental & Water & Water \\
\hline
\end{tabular}

regime remain the ideal configurations for aerosol optical thickness retrieval with $\Delta \tau_{550}^{\mathrm{a}}=0.02$ when available data are from a PRISMA-like sensor. The results confirm that the bright-target in an urban regime and a dark-target in a continental regime, benefiting both by a dark environment, are the best configurations for an accurate $\tau_{550}^{\mathrm{a}}$ retrieval $\left(\Delta \tau_{550}^{\mathrm{a}}=\right.$ 0.02) when data of a PRISMA-like sensor are available.

\section{Conclusions}

This study was based on the idea that hyperspectral visible and near infrared data can be used to provide additional and better spectral information on atmospheric aerosol loadings than multispectral sensors currently in orbit. The study, tailored for retrievals over different surfaces, was based on simulated data and was divided in two main parts: a qualitative analysis performed on an idealized hyperspectral instrument, and a quantitative analysis performed on a PRISMAlike instrument.

The results of the simulation for idealized instrument, which was performed on surfaces with different reflective characteristics, proved that dark surfaces are ideal to retrieve the properties of aerosols, in homogeneous areas, especially under continental aerosol regimes. For urban aerosol condi-
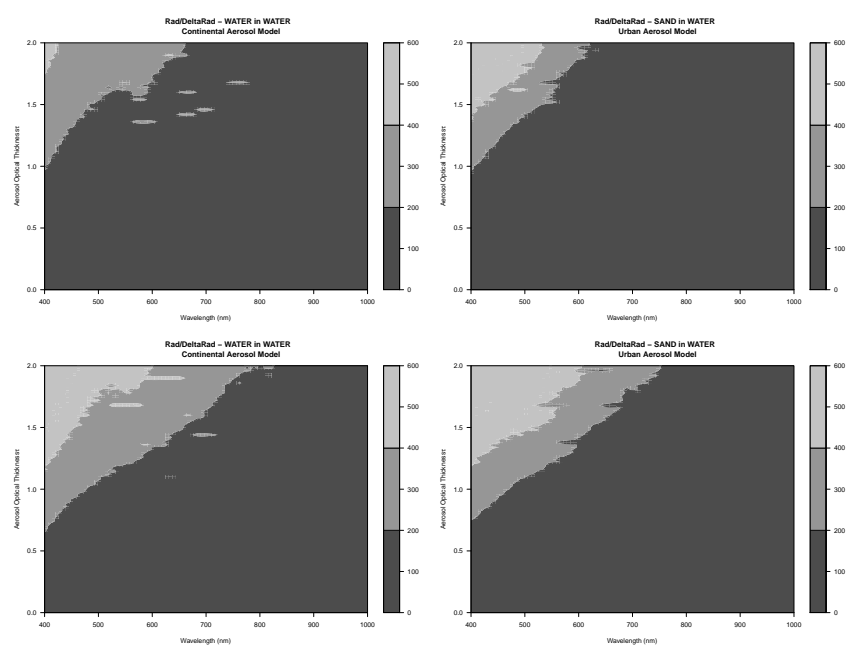

Fig. 9. The $\frac{L(\lambda)}{\Delta L(\lambda)}$ in the PRISMA observed radiance simulated by increasing the aerosol loading, $\tau_{550}^{\mathrm{a}} \in\{0.00-2.00\}$ with $\Delta \tau_{550}^{\mathrm{a}}=$ 0.02 for solar zenith angle $\theta_{\mathrm{s}}=33.97^{\circ}$ (first row) and $\theta_{\mathrm{s}}=66.26^{\circ}$ (second column). The terrestrial surface conditions are dark-target with dark environment in continental regime (first column) and bright-target with dark environment in urban regime (second column).

tions, aerosol retrieval is expected to be more accurate when the target is a bright surface. These results were derived from the radiative components of the observed radiances. For a dark surface, the fraction of solar incident radiation reflected by the surface is negligible with respect to the component diffused by the aerosol. Under these circumstances for aerosol models characterised by significant absorption (as the urban model), the extinction of the radiation happens to be due to both scattering and absorption, and the individual effect of the scattering is attenuated. Consequently, in the presence of absorbing aerosols, as in the urban model, bright surface represents better conditions to retrieve the loading than the dark surface, which is characterised by a smaller diffuse component. Furthermore, the sensitivity of the observed radiance to small variation of the aerosol optical thickness, for bright FOV surface increases when those FOVs are surrounded by dark environment heterogeneous conditions. On the contrary, the sensitivity is reduced if a dark surface is surrounded by bright a environment.

Regarding the PRISMA-like data, the results confirmed that, in general, these kind of sensors would be well suited for retrievals of $\tau_{550}^{\mathrm{a}}$ with an accuracy comparable to the atground measurements $\left(\Delta \tau_{550}^{\mathrm{a}}=0.02\right)$. In particular, results proved that, over heterogeneous regions, bright surface target provides, under the urban aerosol model, better conditions for higher retrieval accuracy compared to the continental regimes. Whereas dark surface targets are less suitable for aerosol retrieval in urban areas and are better suited with for continental aerosolic regimes. In addition, the analysis of the environmental (surroundings) contribution revealed that for 
dark target surfaces, the sensitivity of simulated radiance to small variation of aerosol optical thickness is enhanced by homogeneous environments as compared to heterogeneous environments. Thus, results showed that the adjacency effect, if properly accounted for, improves the sensitivity of the instrument to small variation in aerosol loads and, therefore, it might lead to improvements in the retrieval accuracy of the aerosol optical thickness. Besides the outcome of this study, it is worth emphasizing that the high spatial resolution (Table 3) of PRISMA is expected to enable users to take full advantage of the adjacency effect to improve the accuracy of retrieve aerosol optical thickness at $550 \mathrm{~nm}$, by focusing on an optimal situation (homogeneous or heterogeneous targetssurroundings) for any of the two aerosolic regimes (urban and continental) considered in this study. In particular, the results show the significant role of the environmental contribution on the observed radiance. Both in the case of dark-target in continental regime and bright-target in urban regime, the accuracy of the aerosol optical thickness until $\Delta \tau_{550}^{\mathrm{a}}=0.02$ benefits from a dark-environment.

Presented findings are expected to have important implications for algorithms dedicated to aerosol retrieval from space-borne remote data. This type of remote data provides a new opportunity to jointly process hyperspectral and high spatial resolution $(\approx 30 \mathrm{~m})$ imagery to achieve improved aerosol detection. Further work is required to establish the merits of PRISMA-like observations to derive aerosol loadings by directly using physically-based inversion algorithm on simulated data or, when available, on real data.

Acknowledgements. We thank the referees for their careful reading and helpful comments that greatly improved the manuscript.

Edited by: A. Kokhanovsky

\section{References}

Alakian, A., Marion, R., and Briottet, X.: Remote sensing of aerosol plumes: A semi-analytical model, Appl. Opt., 47, 1851-1866, doi:10.1364/AO.47.001851, 2008.

Barnsley, M., Settle, J., Cutter, M., Lobb, D., and Teston, F.: The PROBA/CHRIS mission, a low-cost small satellite for hyperspectral, multi-angle, observations of the earth surface and atmosphere, IEEE T. Geosci. Remote Se., 42, 1512-1520, 2004.

Bassani, C., Cavalli, R. M., and Pignatti, S.: Aerosol optical retrieval and surface reflectance from airborne remote-sensing data over land, Sensors, 10, 6421-6438, doi:10.3390/s100706421, 2010.

d'Almeida, G. A., Koepke, P., and Shettle, E. P.: Atmospheric aerosols: global climatology and radiative characteristics, A. DEEPAK Publishing, Hampton, Virginia, USA, 1991.

Galeazzi, C., Sacchetti, A., Cisbani, A., and Babini, G.: The PRISMA Program, in: IGARSS 2008, IEEE Int. Geosci. Remote Se., 2008, IV105-IV108, doi:10.1109/IGARSS.2008.4779667, 2008.
Galeazzi, C., Ananasso, C., and Loizzo, R.: The PRISMA Program, in: 6th EARSEL: European Association of Remote Sensing Laboratories, Tel Aviv - Israel, 16-18 March 2009, 1-6, 2009.

Gao, B.-C., Montes, M. J., Davis, C. O., and Goetz, A. F. H.: Atmospheric correction algorithms for hyperspectral remote-sensing data of land and ocean, Remote Sens. Environ., 113, S17-S24, doi:10.1016/j.rse.2007.12.015, 2009.

Goetz, A. F. H., Vane, G., Salomon, J. E., and Rock, B. N.: Imaging spectroscopy for Earth remote-sensing, Science, 228, 11471153, 1985.

Guanter, L., Estellés, V., and Moreno, J.: Spectral calibration and atmospheric correction of ultra-fine spectral and spatial resolution remote sensing data, Application to CASI-1500 data, Remote Sens. Environ., 109, 54-65, doi:10.1016/j.rse.2006.12.005, 2007.

Guanter, L., Segl, K., and Kaufmann, H.: Simulation of Optical Remote-Sensing Scenes With Application to the EnMAP Hyperspectral Mission, IEEE T. Geosci. Remote Se., 47, 2340-2351, doi:10.1109/TGRS.2008.2011616, 2009.

Holben, B., Eck, T., Slutsker, I., Tanré, D., Buis, J., Setzer, A., Vermote, E., Reagan, J., Kaufman, Y., Nakajima, T., Lavenu, F., Jankowiak, I., and Smirnov, A.: AERONET - A federated instrument network and data archive for aerosol characterisation, Remote Sens. Environ., 66, 1-16, doi:10.1016/S00344257(98)00031-5, 1998.

IPCC: The Physical Science Basis, IPCC Fourth Assessment Report: Climate Change 2007 (AR4), Tech. Rep., Intergovernmental Panel on Climate Change, edited by: Solomon, S., Qin, D., Manning, M., Chen, Z., Marquis, M., Averyt, K. B., Tignor, M., and Miller, H. L., 2007.

Kaufman, Y. J., Tanré, D., Gordon, H. R., Nakajima, T., Lenoble, J., Frouin, R., Grassl, H., Herman, B. M., King, M., and Teillet, P. M.: Operational remote-sensing of tropospheric aerosol over land from EOS moderate resolution imaging spectroradiometer, J. Geophys. Res., 102, 17051-17067, doi:10.1029/96JD03988, 1997a.

Kaufman, Y. J., Wald, A., Remer, L. A., Gao, B.-C., Li, R. R., and Flynn, L.: The MODIS $2.1 \mu \mathrm{m}$ channel correlation with visible reflectance for use in remote-sensing of aerosol, IEEE T. Geosci. Remote Se., 35, 1286-1298, doi:10.1109/36.628795, 1997b.

Kaufman, Y. J., Gobron, N., Pinty, B., Widlowski, J.-L., and Verstraete, M. M.: Relationship between surface reflectance in the visible and mid-IR used in MODIS aerosol algorithm - theory, Geophys. Res. Lett., 29, 2116, doi:10.1029/2001GL014492, 2002.

Kaufmann, H., Segl, K., Guanter, L., Hofer, S., Foerster, K.-P., Stuffler, T., Mueller, A., Richter, R., Bach, H., Hostert, P., and Chlebek, C.: Environmental Mapping and Analysis Program (EnMAP) - Recent Advances and Status, in: IGARSS 2008, IEEE Int. Geosci. Remote Se., 2008, IV109-IV112, doi:10.1109/IGARSS.2008.4779668, 2008.

King, M., Kaufman, Y., Tanré, D., and Nakajima, T.: Remote sensing of tropospheric aerosols from space: Past, present, and future, B. Am. Meteorol. Soc., 88, 2229-2259, doi:10.1175/15200477(1999)080;2229:RSOTAF $\dot{~ 2.0 . C O ; 2, ~} 1999$.

Kokhanovsky, A. A.: Aerosol optics: light absorption and scattering by particles in the atmosphere, Praxis Publishing Ltd, Chichester, UK, 2008. 
Kokhanovsky, A. A., Breon, F.-M., Cacciari, A., Carboni, E., Diner, D., Nicolantonio, W. D., Grainger, R. G., Grey, W. M. F., Höller, R., Lee, K.-H., Li, Z., North, P. R. J., Sayer, A. M., Thomas, G. E., and von Hoyningen-Huene, W.: Aerosol remotesensing over land: a comparison of satellite retrievals using different algorithms and instruments, Atmos. Res., 85, 372-394, doi:10.1016/j.atmosres.2007.02.008, 2007.

Kokhanovsky, A. A., Deuzé, J. L., Diner, D. J., Dubovik, O., Ducos, F., Emde, C., Garay, M. J., Grainger, R. G., Heckel, A., Herman, M., Katsev, I. L., Keller, J., Levy, R., North, P. R. J., Prikhach, A. S., Rozanov, V. V., Sayer, A. M., Ota, Y., Tanré, D., Thomas, G. E., and Zege, E. P.: The inter-comparison of major satellite aerosol retrieval algorithms using simulated intensity and polarization characteristics of reflected light, Atmos. Meas. Tech., 3, 909-932, doi:10.5194/amt-3-909-2010, 2010.

Kotchenova, S. Y., Vermote, E. F., Levy, R., and Lyapustin, A.: Radiative transfer codes for atmospheric correction and aerosol retrieval: Intercomparison study, Appl. Opt., 47, 2215-2226, doi:10.1364/AO.47.002215, 2008.
Levy, R. C., Remer, L. A., Kleidman, R. G., Mattoo, S., Ichoku, C., Kahn, R., and Eck, T. F.: Global evaluation of the Collection 5 MODIS dark-target aerosol products over land, Atmos. Chem. Phys., 10, 10399-10420, doi:10.5194/acp-10-10399-2010, 2010.

Seidel, F. C. and Popp, C.: Critical surface albedo and its implications to aerosol remote-sensing, Atmos. Meas. Tech. Discuss., 4, 7725-7750, doi:10.5194/amtd-4-7725-2011, 2011.

Vermote, E. F. and Kotchenova, S.: Atmospheric correction for the monitoring of land surfaces, J. Geophys. Res., 113, 675-686, doi:10.1109/36.581987, 2009.

Vermote, E. F., Tanré, D., Deuzé, J. L., Herman, M., and Morcrette, J. J.: Second simulation of the satellite signal in the solar spectrum, 6S: An overview, IEEE T. Geosci. Remote Se., 35, 675686, doi:10.1109/36.581987, 1997.

Vermote, E. F., Tanré, D., Deuzé, Herman, M., Morcrette, J. J., and Kotchenova, S. Y.: Second Simulation of a Satellite Signal in the Solar Spectrum - Vector (6SV), available at: http: //6s.ltdri.org/6S_code2_thiner_stuff/6s_ltdri_org_manual.htm (last access: 31 May 2012), 2006. 